\title{
1 Not just methods: User expertise explains the variability of outcomes of genome-wide studies
}

Katie E. Lotterhos ${ }^{1}$, Olivier François ${ }^{2,3}$, Michael G.B. Blum ${ }^{2,3 \S}$

${ }^{1}$ Northeastern University Marine Science Center, 430 Nahant Rd., Nahant, MA 01908

$8{ }^{2}$ Univ. Grenoble Alpes, TIMC-IMAG UMR 5525, F-38000 Grenoble, France

$9{ }^{3}$ CNRS, TIMC-IMAG, F-38000 Grenoble, France

10

${ }^{\S}$ Corresponding author Michael Blum Laboratoire TIMC-IMAG, Faculté de Médecine, 38706 La Tronche, France Phone +33456520065

Email: michael.blum@imag.fr

18 data challenge, active learning 


\section{Abstract}

22

23 Genome scan approaches promise to map genomic regions involved in adaptation of

24 individuals to their environment. Outcomes of genome scans have been shown to depend on several factors including the underlying demography, the adaptive scenario, and the software or method used. We took advantage of a pedagogical experiment carried out during a summer school to explore the effect of an unexplored source of variability, which is the degree of user expertise. Participants were asked to analyze three simulated data challenges with methods presented during the summer school. In addition to submitting lists, participants evaluated a priori their level of expertise. We measured the quality of each genome scan analysis by computing a score that depends on false discovery rate and statistical power. In an easy and a difficult challenge, less advanced participants obtained similar scores compared to

34 advanced ones, demonstrating that participants with little background in genome 35 scan methods were able to learn how to use complex software after short 36 introductory tutorials. However, in a challenge of intermediate difficulty, advanced 37 participants obtained better scores. To explain the difference, we introduce a probabilistic model that shows that a larger variation in scores is expected for SNPS of intermediate difficulty of detection. We conclude that practitioners should develop their statistical and computational expertise to follow the development of complex

41 methods. To encourage training, we release the website of the summer school where 42 users can submit lists of candidate loci, which will be scored and compared to the 43 scores obtained by previous users. 
48 The possibility to obtain dense genomic markers at reasonable cost has served the ambition to map genomic regions involved in biological adaptation (Schoville et al. 2012, Savolainen et al. 2013, Haasl and Payseur 2016). Various statistical methods and software have been developed to fulfill this ambition (Rellstab et al. 2015,

52 François et al. 2016). The performances of these methods have been evaluated and 53 compared under various evolutionary scenarios (Narum and Hess 2011, De Mita et 54 al. 2013, De Villemereuil et al. 2014, Lotterhos and Whitlock 2014, Lotterhos and 55 Whitlock 2015). Results obtained with genome scan approaches have been found to depend on the demographic history, on the genetic architecture of adaptation, on sampling design, on statistical software, with interactions between these factors. However, an unexplored source of variability in the application of genome-scan methods is the extent to which different users can achieve different outcomes. In this study, we report the results of a pedagogical experiment carried out at the "Software and Statistical Methods in Population Genomics“ 2015 summer school (SSMPG 2015) to measure how the outcomes of genome scan methods vary among users having different prior levels of expertise.

\section{Material and Methods}

66 The objective of the summer school held in September 2015 was to teach a set of

67 recently developed genome scan methods for the detection of genomic regions

68 involved in local adaptation. The teaching process was based on active learning in

69 which participants were asked to perform data analyses of simulated data using the 70 methods presented (Freeman et al. 2013). Three distinct challenges were proposed 
71 to the participants who had no a priori knowledge of the loci simulated under

72 selection in each challenge. For each challenge, the participants could download

73 simulated genomic data (SNPs) from a dedicated website. They were asked to

74 submit lists of candidate SNPs using the methods presented during the teaching

75 sessions. Lists built from combinations of methods were accepted.

77 The data for the challenges were simulated before the summer school by one

78 instructor, who was the only person who knew how the data were simulated. A total

79 of 48 attendees and the 5 other instructors participated in analyzing the challenges.

80 The datasets contained simulated genotypes for a fictive species. A vast majority of

81 the simulated loci corresponded to selectively neutral alleles while a small fraction of

82 them corresponded to adaptive alleles. The data were simulated by using the 83 computer program NEMO (Guillaume and Rougement 2006).

84

85 The demographic history of the fictive species corresponded to a two-refugia model

86 in a mountain range with three peaks. The species was initially limited to two nunatak

87 (mountain top refuges) during a glaciation period of 3000 generations (Supp Fig 1).

88 At generation 3000, a third nunatak was colonized because of climate warming. At

89 generation 4000, all populations were colonized. For all three challenges, populations

90 had the same demographic history of carrying capacity. However, migration rates or

91 genetic architecture were different between challenges to create increasing difficulty

92 for detecting selection (Supp Fig 2). For all simulations, neutral loci and Quantitative

93 Trait Loci (QTL) were simulated on a genetic map of 6 linkage groups, each $100 \mathrm{cM}$

94 long, with a resolution of $1 \mathrm{cM}$. Simulations of the first challenge assume 12 unlinked

95 QTLs of equal effects on the trait and an island model of migration. Simulations of the 
96 second challenge had the same 12 QTLs of equal effects but migration declined with

97 distance and there was less time until sampling. Simulations of the second and third

98 challenge used the same values of migration rates. In the third challenge, there were

9936 QTLs with effects on the trait as well as some linkage among QTLs. The

100 parameter files used to create the simulations are included as supplementary

101 material (SI Files 1-3).

102

103 Six statistical methods were presented to the participants during 45-minute teaching

104 sessions. The statistical methods were divided into two categories: population

105 differentiation (PD) methods and ecological association (EA) methods. PD methods

106 use allele frequencies to compare single-locus estimates of a population

107 differentiation statistic with their expectation from a null model. EA methods seek for

108 genetic markers significantly correlated with one or several ecological or

109 environmental variables (Rellstab et al. 2015). Methods based on PD methods

110 included HapFlk (Bonhomme et al. 2010, Fariello et al. 2013), OutFLANK (Whitlock

111 and Lotterhos 2015), pcadapt (Duforet-Frebourg et al. 2016), and SelEstim (Vitalis et

112 al. 2014). EA methods included BayPass (Gautier 2015) and LFMM as implemented

113 in the R package LEA (Frichot et al. 2013, Frichot and François 2015).

114

115 For each challenge, participants submitted lists of candidate loci and each list was

116 evaluated by a score. We used an F-score that accounts for sensitivity (or power,

117 POW) and false discovery rate (FDR) as follows (Fawcett 2004):

$$
F=2 \frac{P O W(1-F D R)}{P O W+(1-F D R)}
$$

118 FDR was defined as the proportion of neutral loci in the list and power was defined 119 as the proportion of adaptive loci contained in the list. F-scores range between 0 and 
120 1. The $F$-score was equal to 0 for lists that contained no adaptive loci, and it was

121 equal to 1 if and only if a list matched the list of truly adaptive loci perfectly. Using $F$ -

122 scores, we were able to evaluate the performances of the participants when using a

123 particular method, as well as the variability of their performances in each challenge.

124 At SSMPG 2015, most participants evaluated the number of loci under selection in

125 the simulated datasets quite accurately, although their candidate lists may not have

126 contained true positives only. Under this condition, the F-score was mainly an

127 evaluation of the power (POW) of the participant's approach to detect truly adaptive

128 loci. In this study, our intention is to understand the variability in F-scores of

129 candidate lists submitted by users, rather than variability in the F-scores among the

130 programs employed to detect adaptation.

131

132 For all challenges, participants were able to share expertise and collaborate with 133 each other by building teams. When submitting lists of candidate loci to the website, 134 users (a team or a single individual) had to declare their level of expertise. Two levels 135 of expertise were predefined as "advanced user" or "less-advanced user". For each 136 challenge, a different number of users submitted lists and declared themselves as 137 "advanced users" or "less-advanced users", respectively. In the first challenge, there 138 were 7 advanced and 67 less-advanced submissions, in the second challenge, there 139 were 10 advanced and 56 less-advanced submissions, and in the third challenge, 140 there were 10 advanced and 36 less-advanced submissions.

141

\section{Results}

\section{Difficulty levels of challenges}


144 To provide evidence that the difficulty of correctly identifying adaptive loci increased

145 for each challenge, we evaluated the distribution of scores for each challenge (Figure

146 1). The median of the scores decreased with the challenge number. The median

147 score ( \pm standard deviation) was equal to $0.96( \pm 0.16)$ for the first challenge, $0.40( \pm$

148 0.24) for the second challenge, and $0.31( \pm 0.24)$ for the third challenge. The first

149 case was an easy challenge proposed to all attendees to test software installation

150 and the online submission process. Participants focused their efforts on the

151 challenges 2 and 3 . In the following paragraphs, we report results obtained for the

152 second and third challenges.

153

154 Software usage

155 For the second and third challenges, 124 candidate lists were submitted to the

156 website and 111 submissions were retained after filtering for obvious errors or

157 handling mistakes. The distribution of software usage was almost balanced (Figure

158 2). Three programs (LEA, OutFLANK, pcadapt) represented 60 percent of all

159 submissions. The small bias toward the use of those programs could be explained by

160 the ease to install them as $\mathrm{R}$ packages. The balanced software usage distribution

161 indicated that the users were able to run the 6 programs presented during the tutorial

162 sessions.

163

\section{Variability of scores}

165 Candidate lists that used EA methods obtained higher scores than lists that used PD

166 methods (Figure 3). For both PD and EA approaches and for challenges 2 and 3, the

167 scores were highly variable (standard deviations were in the range $0.18-0.22$ ). We

168 also found high variability of scores when considering the distribution of scores 
169 obtained with each program separately. For the second challenge, standard

170 deviations ranged between 0.01 and 0.34 for the six methods. For 3 programs, the

171 scores had standard deviations around $0.20(+/-0.02)$. For the third challenge,

172 standard deviations were between 0 and 0.22 depending on the software used, and 3

173 programs had a standard deviation in score around $0.20(+/-0.02)$.

174

175 Next we analyzed the scores obtained by each category of users: advanced and

176 less-advanced users. In challenge 2 (intermediate level difficulty), the average score

177 of advanced users was significantly greater than less-advanced users (advanced

178 mean 0.6 , less-advanced mean 0.39 , t-test $P=0.05$ ). In challenge 3 (high level of

179 difficulty), the average score of advanced users was not significantly different than

180 less advanced users (advanced mean 0.35, less-advanced mean 0.30, t-test $\mathrm{P}=$

181 0.45). Similar results were obtained when we considered PD and EA methods

182 separately, but none indicated significant differences between the two groups of 183 users in challenge 3.

184

\section{Discussion}

186 The experiment led during the summer school SSMPG 2015 showed that 187 participants were able to learn how to use complex software for genome scans after 188 short introductory tutorials. In challenge 1 and 3 , less advanced users obtained 189 scores comparable to those of advanced users confirming that a rapid appropriation 190 of software based on complex statistical methods was possible. To encourage 191 submissions, organizers delivered (symbolic) prizes to the two top-ranked user teams 192 for the second and third challenge. Users who won the challenge prizes did not focus 
193 on a particular method, but used combinations of several methods, which is a

194 promising direction to increase statistical power of existing methods.

195

196 Why are the scores variable?

197 In this discussion, we introduce a simple probabilistic model for interpreting the 198 variability of $F$-scores. For each adaptive locus $s$, we assume that a user discovers 199 locus s, i.e., includes locus $s$ in her/his list of candidate loci, with probability $p_{s .}$ We 200 call these quantities the detection probabilities, and we assume that the detection 201 probabilities depend on the level of self-declared expertise of each user. In all 202 challenges, we observed that the users generally evaluated the number of loci under 203 selection rather accurately. Under this condition, the expected value and the variance of the F-scores can be investigated theoretically (results in BOX 1).

205

206

From the theoretical results, we obtain that the variability of scores is directly related 207 to the probabilities of correctly identifying each loci as truly adaptive. We find that the 208 variability of $F$-scores is low when the challenges are either difficult or easy $\left(p_{s}\right.$ are 209 close to 0 or 1 ), and it is maximized for intermediate values of $p_{s}$. For example, let us 210 consider the results of challenge 2 for which the expected score was around .40 and

211 the standard deviation around 0.17 (standard deviations averaged over the different 212 software). We computed the proportion of times each adaptive locus was contained 213 in the submitted lists, and found that the 12 truly adaptive loci spanned a range in 214 their frequency of detection from $\sim 0.1$ to 1.0 (Figure 4). A more complex version of 215 the probabilistic model where probabilities of detection can be different for advanced 216 and non-advanced users (see BOX 1) reproduces the distribution of F-score for 217 challenge 2. Assuming that probabilities of detection are reduced by $40 \%$ for non218 advanced users, that one fourth of the adaptive loci are easily detected by advanced 
219 users $\left(p_{s}=0.9\right)$, one half have intermediate probabilities $\left(p_{s}=0.65\right)$, and one fourth are

220 difficult to detect $\left(p_{s}=0.3\right)$, the probabilistic model generates a distribution of F-scores

221 comparable to the one obtained for challenge 2 (average F-score of 0.41 and 222 standard deviation of 0.16$)$.

223

224 The mathematical results in BOX 1 suggest that the variability of test performances is 225 an inherent characteristic of the data. So, how do we interpret this variability? First, 226 the mathematical results tell us that efforts asked to practitioners in order to reduce 227 the variability of their scores might decrease the overall performance of tests. For example, one might recommend filtering out loci estimated to have small or medium effects on selective traits, and retain only those with strong evidence of selective signals. Such conservative practices would homogenize differences between advanced and less-advanced users and indeed decrease the variability of scores.

232 However this strategy would also result in an increased number of false negative 233 tests, and reduce the overall value of the expected score.

235 The previous example illustrates that the expected $F$-score could be increased if 236 users increase their expertise (and therefore the probability to detect loci of medium 237 effects). Although increasing user expertise is a desirable point, the equations tell us 238 that the variability of scores would not necessarily be decreased. For example, 239 assume there are 12 SNPs of medium effect under selection and the probability of 240 detection of each locus is of $50 \%$ for advanced users and of $5 \%$ for less advanced 241 users. A group with 5\% advanced users and 95\% less-advanced users would have 242 an expected F-score of 0.07 and a standard deviation in F-scores of 0.12 . Now, 243 assume that all users become advanced; although the expected $F$-score of the 
244 advanced group is higher (0.5), the standard deviation in F-scores is also increased,

245 as it is equal to 0.14 . This example shows that reducing the variance of $F$-scores is

246 not a desirable objective, because in some cases increasing user expertise can

247 result in an increase in the variance of $F$-scores.

248

249 The uneven difficulty of detecting adaptive loci, which explains the variability of 250 scores, arises from methods, but also from the decisions made by users regarding 251 model parameters, test calibration and the choice of a false discovery rate in 252 statistical tests. To illustrate how much user decisions can influence the variability of 253 scores, we re-ran two programs, pcadapt and LFMM, on challenges 2 and 3 (using K $254=3$ in both programs). The runs of each program resulted in well-calibrated $p$-values 255 for each data set (François et al. 2016). Then we built lists of candidate loci by using 256 an FDR control algorithm (Benjamini \& Hochberg 1995). The algorithm requires that 257 an expected level of FDR is specified by the user, and uses the expected level of 258 FDR to determine a list of candidates. To simulate users' decisions, we sampled 259 expected levels of FDR according to a beta distribution of mean 0.05 and standard 260 deviation 0.047. The distribution of scores from the created lists had standard 261 deviations of 0.14 and 0.09 for dataset 2 and dataset 3 respectively. These results 262 provide evidence that decisions about how to use the program outputs can generate 263 large variability of scores as observed in Figure 3.

264

\section{Conclusions}

266 The obvious lesson of the SSMPG15 experiment was to promote the usage of 267 powerful statistical methods and simultaneously improve the expertise of their users. 268 The first action is the goal of current methodological developments of genome scans 
269 for selection, which should always be accompanied by clear and practical user

270 guides. The second action requires that practitioners develop their own statistical and

271 computer skills to follow the rapid development of complex methods. To provide a

272 training opportunity, the website containing the data presented during SSMPG 2015

273 as well as additional new datasets is now publicly available (https://ssmpg-

274 challenge.imag.fr/). On the website, users can submit lists of candidate loci that will

275 be scored and compared to the scores obtained by previous users of the website.

276

\section{Box 1: Explaining the variability of $F$-scores.}

278 To explain our results and final recommendations, we introduce a simple probabilistic 279 model for interpreting the variability of $F$-scores. For a given challenge, we assume 280 that the unknown list of truly adaptive loci contains $m$ elements. The values of $m$ 281 were equal to $m=12$ in the first and second challenge and $m=36$ in the third 282 challenge. For each adaptive locus, $s$, we denote by $p_{s}$ the probability (power) that a 283 user discovers locus s, i.e., includes this locus in her/his own list of candidate loci.

284 At SSMPG15, the submitted user lists were approximately of length $m$, meaning that 285 the users correctly evaluated the number of loci under selection in the simulated 286 datasets. Under this hypothesis, we can compute the expected value of the F-score, $287 E[F]$. We obtain that $E[F]$ is equal to $\left(p_{1}+\ldots+p_{m}\right) / m$. Assuming that the tests are 288 independent, the variance of $F$-scores, $\operatorname{Var}(F)$, is equal to $\left(\mathrm{p}_{1}\left(1-\mathrm{p}_{1}\right)+\ldots+\mathrm{p}_{\mathrm{m}}\left(1-\mathrm{p}_{\mathrm{m}}\right)\right) / \mathrm{m}^{2}$. 289 From these results, we obtain that the variability of $F$-scores is directly related to the 290 probabilities to correctly identify each loci as truly adaptive. The variability of $F$-scores 291 is low when the challenges are difficult or easy $\left(p_{s}\right.$ close to 0 or 1$)$, and it is 292 maximized for intermediate values of $p_{s}$. 
293 Accounting for the self-reported expertise of each user, we defined two categories of

294 users: advanced ones, $A$, and less-advanced ones, $A^{\prime}$. In this context, the 295 statements about the expected value and the variance of $F$-scores can be refined as 296 follows. Consider $\pi_{A}$ the proportion of advanced users, and write $1-\pi_{A}$ for less297 advanced users. For a truly adaptive locus $s$, there is a conditional probability, $p_{s A}$, for 298 locus $s$ to be correctly identified by an advanced user, and there is a lower probability, $299 p_{S A}$, for $s$ to be correctly identified by a less-advanced user. The expected value of F300 scores can be computed as follows

$$
E[F]=E[F \mid A] \pi_{A}+E\left[F \mid A^{\prime} \square\right]\left(1-\pi_{A}\right),
$$

302

where the conditional expectation $E[F \mid A]$ is equal to $\left(p_{1 A}+\ldots+p_{m A}\right) / m$, and where the 303 formula for $E\left[F \mid A^{\prime}\right]$ is similar. In other words, the conditional expectations are 304 computed by averaging the conditional probabilities over all adaptive loci. The 305 variance of $F$-scores decomposes as follows

$$
\operatorname{Var}[F]=\operatorname{Var}[F \mid A] \pi_{A}+\operatorname{Var}\left[F \mid A^{\prime}\right]\left(1-\pi_{A}\right)+\left(E[F \mid A]-E\left[F \mid A^{\prime}\right]\right)^{2} \pi_{A}\left(1-\pi_{A}\right),
$$

where $\operatorname{Var}[F \mid A]$ is equal to $\left(p_{1 A}\left(1-p_{1 A}\right)+\ldots+p_{m A}\left(1-p_{m A}\right)\right) / m^{2}$, and the formula for

$308 \operatorname{Var}\left[F \mid A^{\prime}\right]$ is similar. In the discussion, we use the formulae for the mean $E[F]$ and the 309 variance $\operatorname{Var}[\mathrm{F}]$ to measure the effect of filtering strategies and of increasing user expertise on the distribution of $F$-scores.

\section{Acknowledgments}

313 We acknowledge Kevin Caye, Thomas Dias-Alves, and Keurcien Luu for developing 314 the website, and Frédéric Guillaume for providing support with the simulations in 315 NEMO. We would like to thank our colleagues who were instructors during the 316 SSMPG summer school (Mathieu Gautier, Bertrand Servin, and Renaud Vitalis) as 317 well as the attendees of the SSMPG 2015 summer school. 


\section{References}

BENJAMINI Y and HOCHBERG Y (1995) Controlling the false discovery rate: a practical and powerful approach to multiple testing. Journal of the Royal Statistical Society. Series B (Methodological), 57, 289-300.

BONHOMME M, CHEVALET C SERVIN B, et al. (2010). Detecting selection in population trees: the Lewontin and Krakauer test extended. Genetics, 186, 241-262.

DE MITA S, THUILLET A, GAY L, et al. (2013). Detecting selection along environmental gradients: analysis of eight methods and their effectiveness for outbreeding and selfing populations. Molecular Ecology, 22, 1383-1399.

DE VILLEMEREUIL P, FRICHOT É, BAZIN, É, et al. (2014). Genome scan methods against more complex models: when and how much should we trust them?. Molecular Ecology, 23, 2006-2019.

DUFORET-FREBOURG N, LAVAL G, BAZIN E, BLUM MGB. (2016). Detecting genomic signatures of natural selection with principal component analysis: application to the 1000 Genomes data. Molecular Biology and Evolution, 33, 1082-1093.

FARIELLO MI, BOITARD S, NAYA H, et al. (2013) Detecting signatures of selection through haplotype differentiation among hierarchically structured populations. Genetics, 193, 929-941.

FAWCETT T (2004) ROC graphs: Notes and practical considerations for researchers. Machine Learning, 31, 1-38.

FRANÇOIS O, MARTINS H, CAYE K, et al. (2016) Controlling false discoveries in genome scans for selection. Molecular Ecology, 25, 454-469.

FREEMAN S, EDDY SL, MCDONOUGH M, et al. (2014) Active learning increases student performance in science, engineering, and mathematics. Proceedings of the National Academy of Sciences, 111, 8410-8415.

FRICHOT E, SCHOVILLE SD, BOUCHARD G, et al. (2013). Testing for associations between loci and environmental gradients using latent factor mixed models. Molecular Biology and Evolution, 30, 16871699.

FRICHOT E and FRANÇOIS O. (2015) LEA: an R package for Landscape and Ecological Association studies. Methods in Ecology and Evolution, 6, 925-929.

HAASL RJ and PAYSEUR BA. (2016) Fifteen years of genomewide scans for selection: trends, lessons and unaddressed genetic sources of complication. Molecular Ecology, 25, 5-23.

GAUTIER, M. (2015). Genome-Wide Scan for Adaptive Divergence and Association with PopulationSpecific Covariates. Genetics, 201, 1555-1579..

GUILLAUME F and ROUGEMONT J (2006) Nemo: an evolutionary and population genetics programming framework. Bioinformatics, 22, 2556-2557.

LOTTERHOS KE and WHITLOCK MC (2014) Evaluation of demographic history and neutral parameterization on the performance of $F_{S T}$ outlier tests. Molecular Ecology, 2014, 2178-2192.

LOTTERHOS KE and WHITLOCK MC (2015) The relative power of genome scans to detect local adaptation depends on sampling design and statistical method. Molecular Ecology, 24, 1031-1046.

NARUM SR and HESS JE (2011) Comparison of $F_{S T}$ outlier tests for SNP loci under selection. Molecular Ecology Resources, 11, 184-194. 
bioRxiv preprint doi: https://doi.org/10.1101/055046; this version posted May 24, 2016. The copyright holder for this preprint (which was not certified by peer review) is the author/funder, who has granted bioRxiv a license to display the preprint in perpetuity. It is made available under aCC-BY-NC-ND 4.0 International license.

376 RELLSTAB C, GUGERLI F, ECKERT AJ, et al. (2015) A practical guide to environmental association 377 analysis in landscape genomics. Molecular Ecology, 24, 4348-4370.

SAVOLAINEN O, LASCOUX M, and MERILÄ J (2013) Ecological genomics of local adaptation. Nature Reviews Genetics, 14, 807-820.

SCHOVILLE SD, BONIN A, FRANÇOIS O, et al. (2012). Adaptive genetic variation on the landscape: methods and cases. Annual Review of Ecology, Evolution, and Systematics, 43, 23-43.

VITALIS R, GAUTIER M, DAWSON KJ, Beaumont MA (2014). Detecting and measuring selection from gene frequency data. Genetics, 196, 799-817.

WHITLOCK MC and LOTTERHOS KE (2015) Reliable Detection of Loci Responsible for Local Adaptation: Inference of a Null Model through Trimming the Distribution of $F_{S T}$. American Naturalist, 186, S24-S36. 
395 Legends of Figures

396 Figure 1: Distribution of F-scores for the three different data challenges.

397 Figure 2: Distribution of software usage for the second and third challenges.

398 Figure 3: Distribution of F-scores for EA and PD methods. The left and right

399 panel display the results obtained for the second and third dataset, respectively. EA

400 stands for ecological association and PD stands for population differentiation.

401 Figure 4: Probabilities of detection of adaptive loci. For each locus, we counted

402 the proportion of times it was contained in a submitted list. A) Second challenge:

403 probabilities of detection were larger for advanced users than for less-advanced

404 users. B) Third challenge: No obvious differences in detection probabilities when

405 comparing advanced and non-advanced users.

406

407

408

409

410

411

412

413

414

415

416

417

418

419

420

421

422

423
Legends of Supplementary Figures

\section{Supplementary Figure 1}

Schematic temporal evolution of the species range used to simulate data.

Populations 1-3 and populations 15-17 correspond to the two initial glacial refugees. Populations 6,9, and 10 correspond to the summit that was possible to colonize after generation 3,000 . The diameters of the circles increase with increasing effective population size that range from $\mathrm{N}=500$ to $\mathrm{N}=2500$. Colors on the landscape represent the phenotypic optimum for the trait, ranging from an optimum of -3 (dark green) to 3 (white).

Supplementary Figure 2

Parameter settings used in the simulations of the three different challenges. 


\section{Software Usage}

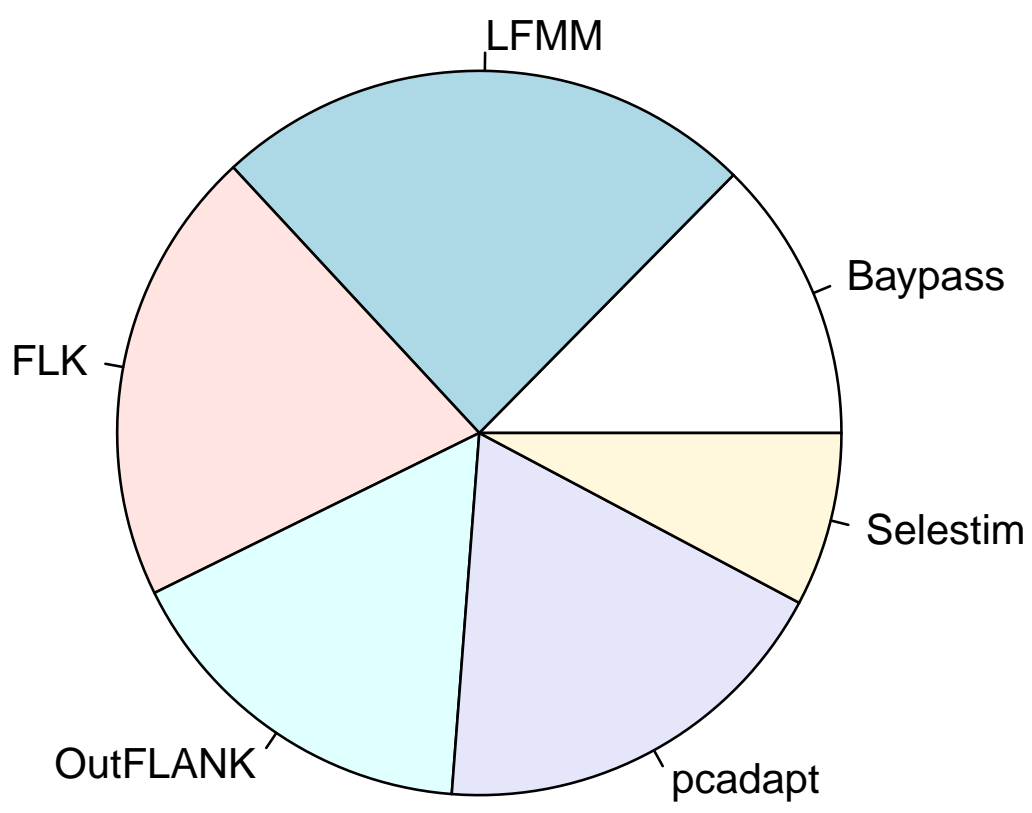


Challenge 2

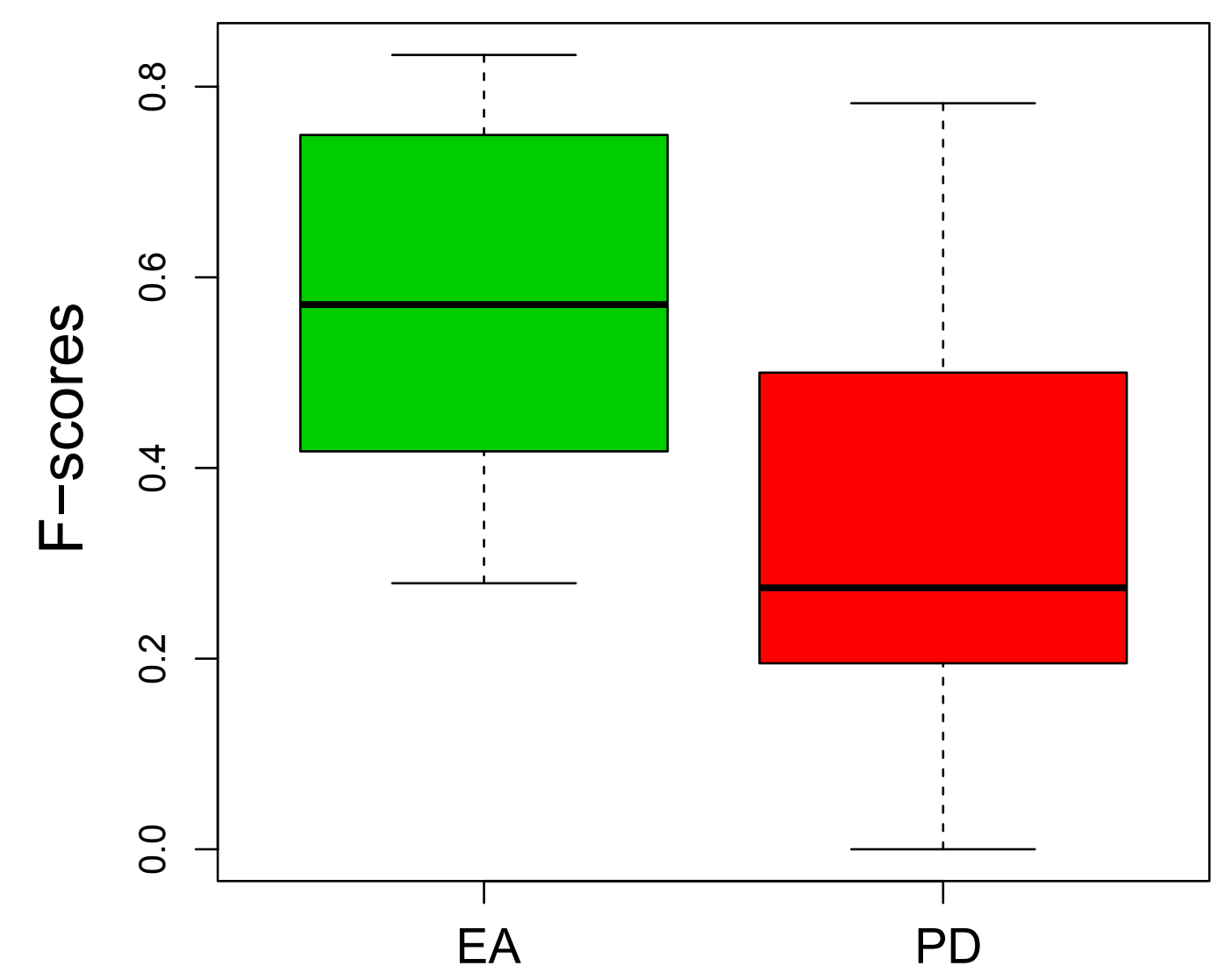

Challenge 3

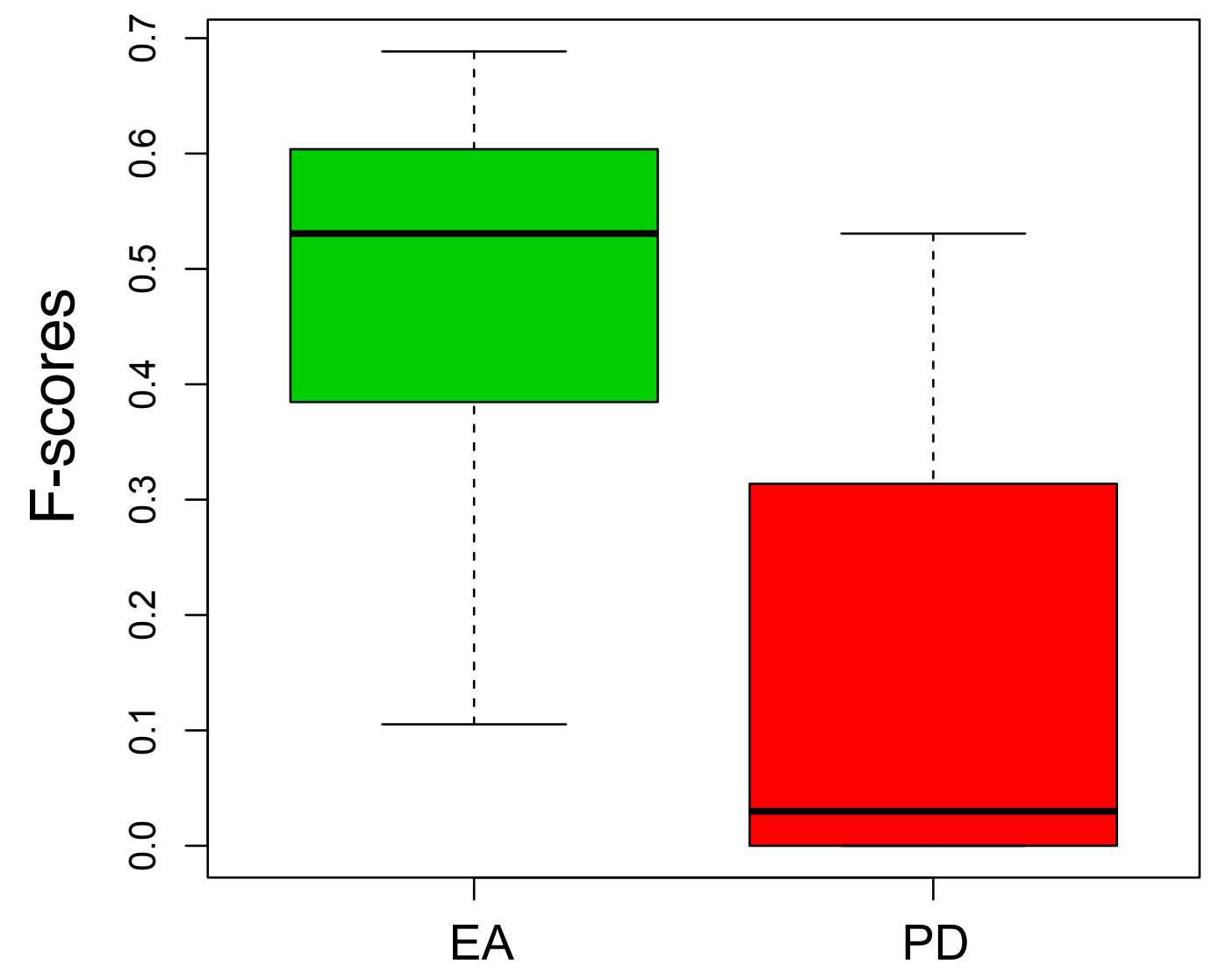




\section{A}

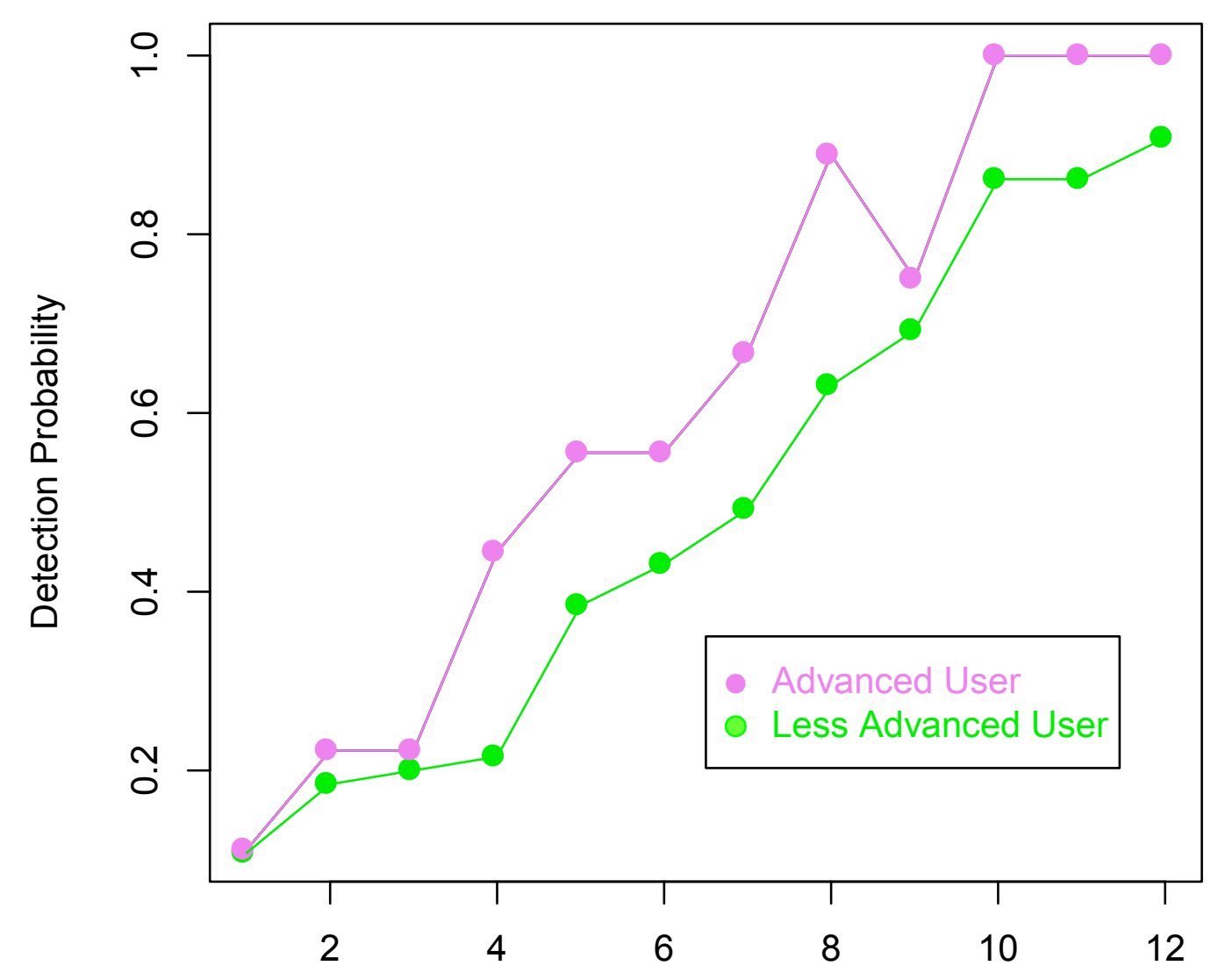

Truly Adaptive Locus
B

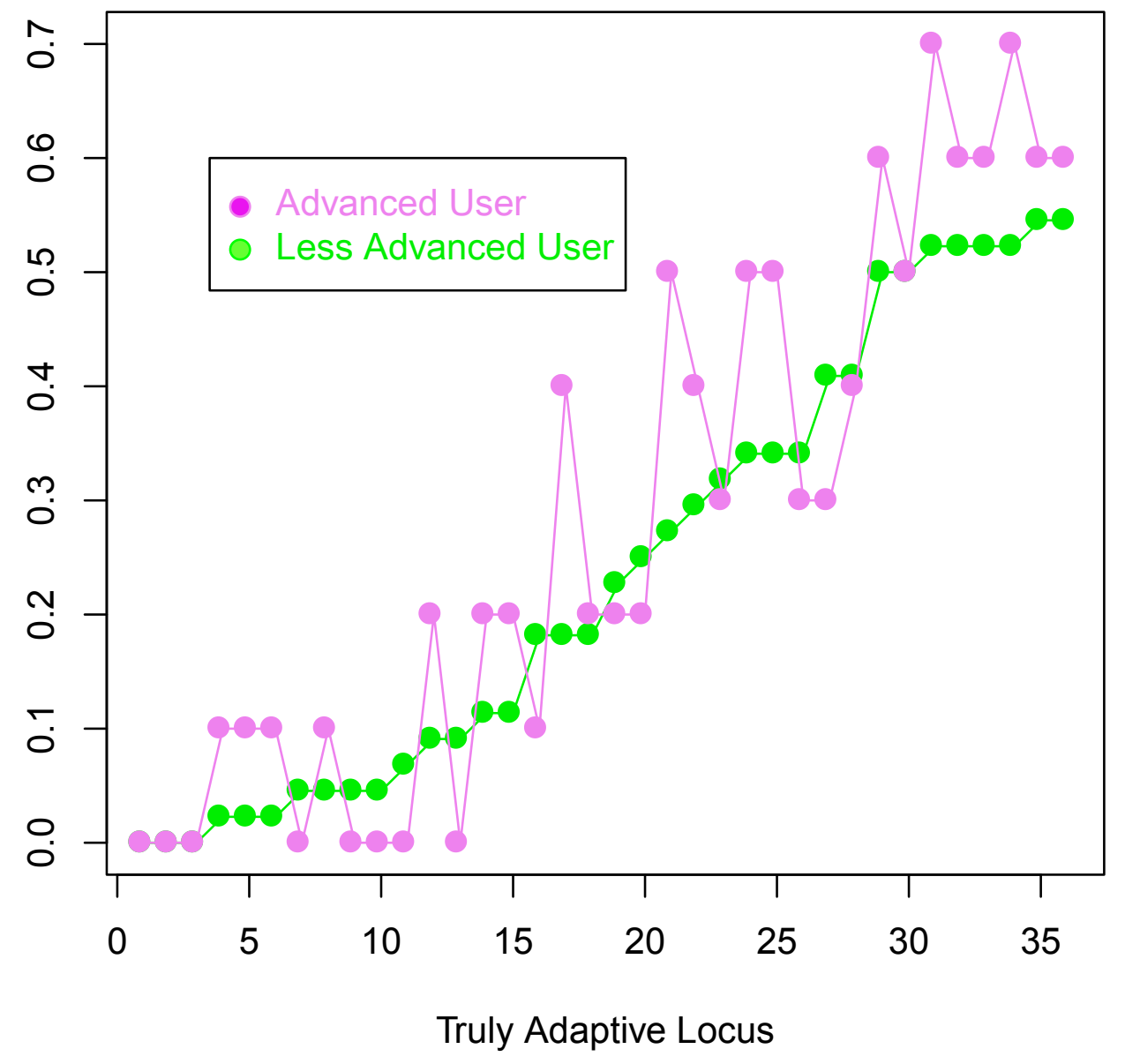

